\title{
Educación para el bilingüismo y aprendizaje de maestros: comprensión del desempeño auténtico en la acción de cambiar prácticas pedagógicas
}

\author{
Education for Bilingualism and Teacher Learning: \\ Understanding Authentic Performances as \\ Pedagogical Practice Changes
}

Claudia Lucía Ordóñez ${ }^{1}$

Resumen

Buscando el bilingüismo en estudiantes de un colegio privado de ciudad intermedia en Colombia, un contexto totalmente monolingüe, diseñé y puse en práctica -con un grupo de profesionales de la institución- un currículo basado en desempeños auténticos comunicativos ${ }^{2}$, que conecta desarrollos en español e inglés. Dirigí paralelamente una investigación cualitativa sobre su impacto ${ }^{3}$ durante el primer año de su uso, que revela grandes cambios en los ambientes de aprendizaje de lengua en el colegio y una diversidad de niveles de comprensión de este principio fundante del currículo entre los maestros. Esta diversidad de comprensión tiene la particularidad de provenir casi exclusivamente del desempeño auténtico pedagógico, de cambiar radicalmente sus concepciones y prácticas de aula.

\section{Palabras clave}

Educación para el bilingüismo, desempeño auténtico para aprender, bilingüismo en contexto monolingüe, currículo bilingüe.

\section{Abstract}

In order to achieve bilingualism in the students of a private school in a medium-sized city in Colombia, a totally monolingual context, I and a group of professionals in the institution designed a curriculum based on authentic communicative performances, connecting developments in Spanish and English. I directed parallel qualitative research on its impact during the first year of its use, revealing great changes in the language learning environments in the school and a variety of levels of understanding of this founding principle among the teachers. This diversity in understanding comes almost exclusively from the authentic pedagogical performance of radically changing conceptions and practices of what has to happen in the classroom.

\section{Keywords}

Education for bilingualism, authentic performance for learning, bilingualism in a monolingual context, bilingual curriculum.

Artículo recibido el 23 de octubre de 2011 y aprobado el 19 de julio de 2012

1 Universidad Nacional de Colombia, Bogotá, Colombia. Correo electrónico: clordonezo@unal.edu.co

2 Un desempeño auténtico es un proceso, acción o producto que se realiza en un contexto determinado para lograr propósitos auténticos en el mundo real. Se refiere a lo que hacen las personas que usan sus comprensiones en la vida diaria, la vida profesional o la actividad experta. En lengua, un desempeño auténtico persigue propósitos de comunicación entre interlocutores y/o escritores y lectores (Ordóñez, 2010).

3 Este artículo es uno de los resultados de la investigación "Evaluación de la puesta en práctica de un currículo conectado de español e inglés en un colegio bilingüe", llevada a cabo con financiación de la Facultad de Ciencias Humanas de la Universidad Nacional de Colombia, dentro de la convocatoria Orlando Fals Borda de 2009. Participaron todos los coordinadores y profesores de los departamentos de español e inglés de un colegio privado, bilingüe, de una ciudad intermedia colombiana. 


\section{Educación para el bilingüismo en el contexto colombiano}

En la introducción a las Memorias del II Simposio de Bilingüismo y Educación Bilingüe en América Latina (Bilinglatam II, 2007), que compilé después del evento realizado en la Universidad de los Andes en 2006, presentaba el término "educación para el bilingüismo" de la siguiente manera:

.... tanto desde las medidas políticas como desde las estrategias prácticas, que utilizamos para formalizar el logro del bilingüismo desde lo educativo, tendemos a olvidar que la lengua materna necesita estímulo y desarrollo especiales en la escuela; a olvidar que su ocurrencia social no es suficiente para asegurar su desarrollo y enriquecimiento permanentes, que son funciones naturales de la escuela, desde los nuevos mundos de aprendizaje y comunicación que abre para los niños y jóvenes. Sea ésta la oportunidad de introducir la idea de la educación para el verdadero bilingüismo, más allá de la de la educación bilingüe que manejamos, hasta ahora, con poca o incompleta eficacia. (Ordóñez, 2007)

$\mathrm{Y}$ es que necesitamos una nueva manera de referirnos a lo que debe pasar en la escuela para que nuestros niños y jóvenes lleguen a ser bilingües. En nuestra limitada experiencia colombiana en la búsqueda del mejor aprendizaje posible de lenguas extranjeras, las instituciones que consideramos logran mayor éxito son los colegios que denominamos bilingües, generalmente colegios privados de élite en nuestras grandes ciudades que tienden a concentrarse en el desarrollo de la lengua extranjera y a dejar de lado el español (De Mejía, Ordóñez y Fonseca, 2006). Este pierde importancia y tiempo de uso y su desarrollo parece darse por descontado, sin dedicarle mayor esfuerzo pedagógico. Estos colegios han adoptado modelos de educación bilingüe desarrollados para contextos ajenos a los nuestros. Muchos siguen los programas y prácticas de la inmersión canadiense o, inclusive, programas, políticas y formas de acreditación de colegios monolingües de países europeos o de los Estados Unidos (De Mejía et al, 2006). Lo que hacen, básicamente, es crear ambientes artificiales de uso de la lengua extranjera de interés, por medio de cuatro acciones básicas: la introducción lo más temprano posible de la lengua extranjera, normalmente en el nivel de preescolar y a menudo en programas de inmersión parcial o total; el aprendizaje de áreas académicas distintas a la lengua misma en lengua extranjera; la contratación de profesores extranjeros de habla nativa; y un esfuerzo grande para que la mayoría de gente hable en lengua extranjera en el colegio, dentro y fuera de las clases.

Desde luego, estas decisiones políticas son consistentes con el sentido común: si la lengua extranjera no se usa en el contexto real, hay que darle la mayor cantidad de dedicación, tiempo y calidad posibles en el colegio. Pero un conocimiento más especializado de los procesos de adquisición lingüística permite consideraciones diferentes, así como el conocimiento procedente de la investigación. La poquísima investigación que hemos realizado en estos colegios en Colombia, por ejemplo, ha producido alguna evidencia inicial de que la producción en español de los alumnos de estos colegios presenta una exagerada influencia de las características pragmáticas del inglés y revela una calidad empobrecida de sus habilidades de narración en lengua materna, al compararlas con las de estudiantes de colegios monolingües en español (Ordóñez, 2000, 2004a, 2005). Darle una exagerada importancia a la lengua extranjera, introducirla demasiado temprano en el desarrollo lingüístico del niño y reducir considerablemente el tiempo de estimulación y uso de la lengua materna, pueden no ser las formas más eficaces de lograr un verdadero bilingüismo en contextos mayoritariamente monolingües como los nuestros, en los que la lengua materna debería lograr un desarrollo avanzadísimo.

Pero no es fácil pensar así. Nuestro monolingüismo es el principal problema que enfrentamos para el aprendizaje de lenguas extranjeras, en tiempos en los que resulta difícil discutir la importancia que tiene para la vida saber otro idioma, especialmente el inglés (Ordóñez, 2008). Dos hechos sobre la adquisición de una lengua se sustentan consistentemente en la investigación: todas las personas pueden adquirir o aprender una o varias lenguas, pero el éxito de 
esa adquisición o aprendizaje está condicionado a la necesidad que tengan quienes aprenden, de usar esa(s) lengua(s) en la comunicación auténtica (e.g. Snow, 2007). Esto se debe a que son las necesidades y las exigencias pragmáticas de la comunicación, o sea las características, relaciones y propósitos de quienes se comunican y las condiciones contextuales en las que lo hacen, las que motivan e impulsan el aprendizaje de una lengua, como ocurre en el proceso natural de desarrollo de la lengua materna en la infancia (e.g. Ninio y Snow, 1996). Esto quiere decir que el hecho de que nuestros niños y jóvenes escolares no necesiten usar el inglés para funcionar en su vida diaria, hace especialmente difícil para ellos encontrar sentido en aprenderlo, aunque las políticas educativas nacionales establezcan que así debe ser.

Por otro lado, aunque no se espera que un hablante bilingüe exhiba habilidades iguales a las de dos personas monolingües, tampoco se justifica que en ambientes sociolingüísticos monolingües las habilidades en lengua materna se vean reducidas o limitadas, sin necesidad, en la búsqueda del bilingüismo. Y es que hay otro hecho de la adquisición o el aprendizaje de lengua que parece ser desconocido en nuestra educación bilingüe: después de los 4 o 5 años de edad, el estímulo principal para el desarrollo del lenguaje es la experiencia escolar, por varias razones: al entrar al colegio el niño se enfrenta a la necesidad de comunicarse con una diversidad de personas nuevas en una diversidad de situaciones comunicativas nuevas, y comienza a conocer nuevos temas y tipos de discurso que se asocian, por ejemplo, con las diferentes áreas académicas. Además, inicia la lectura y la escritura, que constituyen la mayor fuente de expansión lingüística posible (e.g. Barriga-Villanueva, 1998, 2002). Esto, desde luego, aplica primero que todo para la lengua materna, y debe hacernos reflexionar sobre la riqueza que debe rodear su desarrollo formal y sobre la necesidad de que esa riqueza se mantenga cuando se sume en la escuela el propósito del bilingüismo.

La dificultad en la que se constituye nuestro contexto sociolingüístico monolingüe para el aprendizaje de lenguas extranjeras, varía en grados de intensidad en las muy diferentes circunstancias en las que viven las comunidades en un país tan diverso como Colombia. Un ejemplo sencillo son nuestras ciudades pequeñas, en donde las lenguas extranjeras están aún más lejanas de la cotidianidad de los niños y jóvenes que en las grandes ciudades capitales. En estas últimas resulta un poco más fácil encontrar extranjeros y sitios en los que, al menos a nivel de actividades turísticas y comerciales, se encuentran en uso otras lenguas. También es más fácil encontrar librerías que venden libros en otras lenguas, bibliotecas que los contienen y situaciones de trabajo en las que se necesitan, por ejemplo. Por eso mismo es más fácil encontrar en las ciudades grandes colombianos que saben otras lenguas y eventualmente las utilizan. Pero es en una ciudad intermedia colombiana que se localiza el colegio objeto de este artículo.

\section{Un currículo específico para un contexto específico}

El colegio atiende niños y niñas de estrato socioeconómico alto, y había hecho un esfuerzo continuado durante alrededor de 15 años para alcanzar el deseado bilingüismo por medio de las cuatro acciones indicadas arriba, sin mucho éxito. Parecía que los estudiantes no hablaban inglés fácil ni naturalmente, que no lo hacían por iniciativa propia ni con gusto, y que en vez de inglés utilizaban una extraña lengua híbrida, mejor descrita como español con palabras inglesas. A menudo se observaba falta de interés e inclusive verdadera resistencia entre los niños y jóvenes escolares hacia el uso de esta lengua. Como posibles razones se pensaba en fallas de formación en los profesores colombianos y en la necesidad de intensificar las cuatro medidas que hacen que un colegio pueda llamarse bilingüe.

La administración del colegio buscó mi asesoría en la solución de estos problemas y demostró gran apertura a posibilidades completamente diferentes. Normalmente no se piensa que el problema esté más bien en el contexto, en la poquísima justificación y el poquísimo sentido que encuentran los niños y jóvenes en el uso del inglés en sus vidas. Y aún menos se piensa en soluciones que se basen en una atención 
mucho mayor al desarrollo crecientemente complejo de las habilidades comunicativas en español, y en el establecimiento de conexiones entre este desarrollo y el de la lengua extranjera; o sea, en el manejo de un verdadero bilingüismo en el proceso de buscar el bilingüismo, en una verdadera educación para el bilingüismo.

La institución aceptó emprender el amplio cambio curricular necesario para ensayar estas ideas, que no se encuentran en ejercicio en el colegio bilingüe modelo. Así, durante el año académico 2007, formé un grupo de estudio conformado por los coordinadores de las dos áreas de lengua y algunos profesores de inglés y español cuyo tiempo pudo liberar el colegio, y emprendimos un trabajo pionero de diseño curricular. Tuvimos discusiones sobre la situación del desarrollo del área de lenguaje en el colegio y el currículo que se utilizaba, y lectura crítica de algunos pocos documentos teóricos que nos ayudaran a considerar posibilidades diferentes de desarrollo lingüístico en general, desarrollo del bilingüismo y manejo del aprendizaje de los niños.

Logramos consenso acerca de la conveniencia de emprender cambios y el diseño de algunas actividades ejemplares para ensayar en las clases de las dos lenguas. Los maestros de español e inglés de todos los grados las ensayaron juiciosamente, junto con otras que diseñaron individualmente $o$ en grupos por nivel, durante el año 2008, intercalándolas dentro de un currículo tradicional conformado por logros limitados mecánicamente por los tiempos establecidos en el colegio.

Al final de este año convertí la experiencia colectiva en un currículo con las siguientes características: aplica principios constructivistas del aprendizaje humano, como el que postula que la acción auténtica en el mundo para aprender, se conecta con la teoría lingüística comunicativa para producir los "desempeños auténticos comunicativos", base de su diseño; es realmente bilingüe, debido a que reconoce la importancia que da el constructivismo al aprendizaje previo en la construcción de todo conocimiento nuevo y, por consiguiente, se sustenta en la adquisición de la primera lengua; y cubre tres áreas de desarrollo comunicativo: la de la comunicación diaria y de los medios (radio, TV, periódicos, revistas, la Internet, etc.), la de la aproximación al conocimiento de las diferentes disciplinas académicas y la de la expresión artística y cultural (Ordóñez, Bonilla, Arias, Casallas, Barrero y Lastra, 2008).

\section{La teoría plasmada en las características del currículo}

Las características del currículo son consistentes con cuatro principios constructivistas provenientes de diferentes pensadores, quienes han aportado a la compleja construcción que es hoy en día, no la pedagogía constructivista, sino la teoría que describe el aprendizaje humano. El primero habla del aprendizaje en la acción, pero no cualquier acción. Piaget (1970) se refiere a acciones de manipulación de los elementos del ambiente; Vygotsky (1978) a acciones sociales con compañeros y tutores de niveles diferentes de desarrollo y conocimiento, mediadas por el lenguaje; Perkins (1998) a acciones que lleven realmente a la comprensión de lo que se aprende; Dewey (1945) y la teoría de la cognición situada (Brown, Collins y Duguid, 1989; Díaz Barriga, 2003) a la acción tal como ocurre auténticamente en la vida real. Desde aquí, y conectando con el hecho de que la acción auténtica con el lenguaje es siempre el uso del pensamiento y la emoción en la comunicación con otros, nuestra construcción curricular se basa en "desempeños auténticos" (Ordóñez, 2010), en este caso comunicativos.

El reconocimiento de la acción con otros como un fenómeno natural de la comunicación en el mundo real se refuerza con el segundo principio constructivista, de naturaleza sociocultural y establecido inicialmente por Vygotsky (1978). Postula el aprendizaje individual como motor del desarrollo del pensamiento, pero lo condiciona al estímulo de la interacción con otros. Por esta razón, el currículo orienta la forma como debe ocurrir el aprendizaje dentro y fuera de los salones de clase hacia el trabajo en colaboración para aprender. Ya el grupo de diseño vivía la necesidad trabajar en colaboración dentro y entre las áreas de lengua para lograr los cambios deseados. 
El tercer principio, poco considerado en diseño curricular en la educación formal, indica que todo aprendizaje ocurre naturalmente como proceso (Piaget, 1970). Por esto el currículo no ordena que lo que se aprende empiece y termine en períodos determinados de tiempo. En cambio, lo que vale la pena aprender aparece y reaparece repetidamente en diferentes grados y en desempeños parecidos que deben adaptarse a los diferentes intereses y contextos de los niños según su edad. Así, el lenguaje que exigen aumenta paulatinamente en complejidad y todo el repertorio de lenguaje que se desarrolla se mantiene en uso permanente. Esto corresponde a un currículo de naturaleza cíclica (Dewey, 1948), cuyo propósito no es diferenciar períodos de enseñanza o grados escolares según contenidos académicos, sino orientar procesos de aprendizaje.

El cuarto principio contribuye a esta característica cíclica del currículo, pues basa todo aprendizaje nuevo sobre el conocimiento ya construido (Piaget, 1970; Ausubel, 1968). La aplicación de este principio hace que en el currículo se conecten constantemente los desempeños comunicativos y el lenguaje, ya introducidos y utilizados, con los que se presentan por primera vez, lo cual se plasma también en ese aparecer y reaparecer permanente de desempeños que se han realizado anteriormente o que se relacionan fácilmente con desempeños anteriores. Este principio también sustenta la construcción de habilidades en la lengua extranjera sobre las que ya el niño y el joven han desarrollado en su primera lengua, característica que define el currículo como bilingüe.

Para buscar un verdadero bilingüismo, entonces, el currículo indica que todo desarrollo comunicativo se trabaja primero en español; que se ponen en uso las dos lenguas en desempeños de comunicación similares en los mismos o en diferentes niveles escolares; y que, siempre que sea posible y natural, los alumnos usan las dos lenguas en el desarrollo del mismo desempeño. En todos los casos en que las dos lenguas entran en contacto, el currículo obliga a los maestros a trabajar juntos en la planeación y la puesta en práctica de la instrucción (e.g. cuando trabajan con sus alumnos la búsqueda y uso de información en investigaciones bibliográficas; cuando planean con ellos entrevistas para hablantes de una lengua u otra en investigaciones periodísticas, personales o empíricas; cuando desarrollan programas de radio o televisión, noticieros y periódicos bilingües; etc.). Y los obliga a no temerle a sus vacíos de conocimiento o de habilidad en una lengua o la otra, o a una posible interferencia entre las dos: los maestros deben trabajar en equipo para compensar los primeros y ayudar a los estudiantes a comparar las dos lenguas para entender y evitar la segunda. Porque el verdadero bilingüe consecutivo tiene y usa naturalmente el conocimiento de su primera lengua para aprender y usar una nueva, pero debe poder reconocer las diferencias entre las dos. No logrará esto nunca si se evita el uso de la primera lengua para, supuestamente, favorecer el de la extranjera.

Finalmente, las tres áreas de desarrollo comunicativo que toca el currículo, vida diaria y mediática, académica y estético-cultural, no son desconocidas para ningún maestro de lengua. Sin embargo, una o varias pueden resultar olvidadas o al menos descuidadas en sus clases. Por ejemplo, muchos maestros trabajan la lengua solamente a partir de la literatura, con lo cual se limitan a unos tipos de discurso y olvidan la importancia que tiene el desarrollo de habilidades para el manejo de discursos diferentes. Los diversos tipos de discursos académicos, por ejemplo, normalmente se dejan en manos de los maestros de otras áreas, a menudo poco conscientes del funcionamiento pragmático del lenguaje en ellos. El currículo recuerda permanentemente a los maestros que deben trabajar desempeños comunicativos en las tres áreas y conectarlos, en la medida de lo posible.

\section{La autenticidad}

La autenticidad como cualidad de las actividades de aprendizaje, esa que se constituye en característica básica del currículo objeto de este artículo, ocurre rara vez en los salones de clase. Se refiere a aquello que acerca lo que se hace en ellos para aprender, a la realidad de lo que viven las personas todos los días al compartir o no cosas, lugares, actividades, trabajos, objetivos y propósitos en comunidades 
construidas por ellas mismas y conformadas por gente diversa que entra en relación. Las actividades de aula solo llegan, eventualmente, a la simulación de contextos en los que se aplican conocimientos que se imaginan ya comprendidos, sin mayor reflexión sobre su uso ni solución de problemáticas reales, indican Brown et al (1989) y Díaz Barriga (2003). Lave (1997) discute también la falta de autenticidad de las actividades escolares, señala su inefectividad e indica que mientras los problemas propuestos a quienes aprenden partan de los maestros, el currículo o los textos, sus desempeños no dejarán de ser simples improvisaciones.

Perrone (1998) ubica el origen de la idea de que el aprendizaje escolar ocurra en la experiencia y en y para contextos que trasciendan la escuela misma, o sea en la autenticidad, en las reformas educativas progresistas de principios del siglo XX en los Estados Unidos. Luego Dewey (1945), Bruner (1960) y Piaget (1999) abogaron de distintas formas por la necesidad de una pedagogía que integrara el contenido escolar, las actividades cotidianas del niño y su experiencia, que le propusiera problemas de aprendizaje no artificiales y que desarrollara en él destrezas que pudiera utilizar en actividades fuera del aula. Más recientemente, Lave (1997) y StoneWiske (1998) indican que la apropiación de los problemas y la comprensión están estrechamente relacionadas con el sentido personal con que un aprendiz construye conocimiento a partir de su propia experiencia y trabajo intelectual. Y Savery y Duffy (1996) consideran que además de la conexión personal con lo que se aprende, se requiere que lo que se haga produzca procesos cognoscitivos similares a los de quienes actúan en la vida real.

La definición más clara del desempeño auténtico como forma de aprender, proviene de las evidencias que han producido investigaciones en las cuales se ha observado que el aprendizaje no puede desligarse del contexto donde ocurre: la cognición es "situada", indican Brown et al (1989). Partiendo de esta idea, concluyen que son las prácticas ordinarias de una comunidad o cultura, o sea las actividades auténticas, las que permiten a quienes aprenden acercarse al sentido y al propósito del conocimiento en el mundo real. De allí, Collins (citado en Brill, 2001) pasa a afirmar que las actividades escolares situadas en contextos específicos y acompañadas de apoyo, permiten aprender en qué circunstancias reales se emplea el conocimiento y cuáles son las implicaciones de su uso, de modo que los estudiantes puedan solucionar problemas en nuevas situaciones.

El "constructivismo del desempeño" (Perkins, 1998, p. 57) toma ideas de todos estos pensadores cuando define la comprensión, sinónimo de aprendizaje, como la habilidad para pensar y actuar de manera flexible, o sea en diferentes contextos, a partir de lo que se sabe. Perkins (1998) indica que quienes aprenden deben desarrollar conocimiento acerca de la utilidad de lo que aprenden y Boix-Mansilla y Gardner (1998), que deben usar el conocimiento para construirlo. Perkins (1993) y Gardner (2004) también hablan específicamente de desempeños escolares en los que se usen formas de pensamiento y acción propias de los diferentes campos disciplinares, de modo que quienes aprenden se acerquen a cómo procede un experto en la comprensión del mundo. Ya Brown et al (1989) habían señalado que las comunidades académicas son grupos culturales específicos en los que se debe "situar" el aprendizaje. Ordóñez (2004b), por su parte, orienta el diseño pedagógico de desempeños auténticos, indicando que provienen del análisis de problemas y modos de pensar de las personas comunes que actúan en el mundo con comprensión de lo que saben, y de especialistas en diferentes disciplinas y profesiones que utilizan su conocimiento, igualmente, en el mundo real. La autenticidad se presenta, pues, hoy en día, como característica indispensable de las actividades que se diseñan para que los estudiantes de cualquier edad aprendan.

En la pedagogía del lenguaje, el desarrollo de destrezas relacionadas con su uso en el mundo real ha tenido que ver con el contacto con materiales escritos y orales auténticos, desde tiempos históricos remotos (Insuasty, 2008). Pero además, en los más de 30 años de desarrollo y uso pedagógico del enfoque comunicativo de análisis del lenguaje, la lingüística aplicada ha aportado a este campo el conocimiento que permite la enseñanza y el aprendizaje del lenguaje 
como verdadera herramienta de comunicación. El aprendizaje de lengua debe ocurrir en la comunicación auténtica, indica la lingüística aplicada, ya que las formas lingüísticas dan y adquieren significado solo en ella, y es la pragmática la que permite la interpretación completa de los significados que se intercambian auténticamente, tanto en el medio oral como en el escrito. La pragmática analiza las condiciones que definen los actos de comunicación: quién envía y quién recibe un mensaje, desde qué posiciones y con qué propósitos lo hacen, qué relaciones hay entre ellos y en qué contexto lo producen (e.g. Widdowson, 1978; Van Lier, 2005). Y además la pragmática, en conexión con el constructivismo vygotskiano (Vygotsky, 1978), influye hoy en los estudios sobre la adquisición natural del lenguaje, que a su vez influyen más y más en las decisiones sobre aprendizaje formal de cualquier lengua, develando la manera como los infantes y los niños desarrollan sus habilidades lingüísticas en la comunicación auténtica con quienes los rodean, gracias a estímulos de naturaleza pragmática (Ninio y Snow, 1996).

\section{Un difícil trabajo para los maestros}

Las circunstancias de la puesta en práctica del currículo hicieron que el trabajo de los maestros participantes fuera especialmente difícil $y$, por consiguiente, que sus logros tengan un extraordinario valor. En primer lugar, convencida de que ninguna cantidad de preparación les hubiera dado suficiente seguridad para afrontar los cambios enormes que exigía el currículo, y fiel a la teoría constructivista que guió su diseño, simplemente estimulé a los maestros a emprender la construcción de la comprensión de lo que debían hacer en la acción directa: debieron lanzarse a ensayar los desempeños desarrollados por el grupo de diseño y a diseñar los propios, usando su conocimiento y experiencia naturales acerca de la comunicación. El éxito del currículo centrado en la autenticidad dependió enteramente, entonces, de la habilidad de los maestros para analizar sus propias experiencias de comunicación y las de otros, de modo que pudieran diseñar desempeños de aprendizaje para sus clases que correspondieran a situaciones, acciones, procesos y productos comunicativos de la vida real.

En segundo lugar, los maestros debían analizar en detalle las características, conocimientos e intereses de sus estudiantes y los contextos en los que se movían, porque el nivel de autenticidad de los desempeños que pueden diseñarse varía según esos contextos, los niveles de desarrollo psicológico $\mathrm{y}$ cognoscitivo de los alumnos, sus inclinaciones $\mathrm{y}$ las posibilidades de uso de recursos que se tengan al alcance. Había que moverse desde lo conocido e interesante para los niños y sus desarrollos en español, hacia el uso de esos desarrollos e intereses también en inglés y, finalmente, hacia el interés y la autenticidad en inglés, en la medida de lo posible. Los maestros debían estar siempre preparados para permitir la participación de los estudiantes en las decisiones de clase y usar sus ideas, y listos para conectarse con maestros de otras áreas cuando las ideas obligaran a relacionar temas de trabajo, como ocurre en la vida real cuando se busca comprender: se usa el conocimiento y no se separan las materias.

Una tercera dificultad fue que para permitir la adaptación a grupos específicos de estudiantes, el currículo solamente enuncia grandes desempeños, de manera muy general. Fue labor de los maestros interpretar estos enunciados generales y convertirlos en desempeños auténticos, comunicativos, específicos y adecuados con y para sus alumnos. Un ejemplo de esa generalidad puede verse en el siguiente extracto de lo que indica el currículo para español y para inglés de $3^{\circ}$ y $4^{\circ}$ de primaria, en diferentes secciones y para las áreas de comunicación diaria y mediática:

- Hacer y contestar preguntas sobre los propios intereses y observaciones.

- Leer textos instructivos (recetas y manualidades) y seguir las instrucciones.

- Dar instrucciones sobre actividades diversas para que otros puedan seguirlas.

- Leer noticias de periódicos y revistas infantiles y de adultos y ver noticieros de televisión, infantiles y para adultos, y discutirlos.

- Expresar sentimientos y emociones en mensajes personales y en propagandas, con apoyo gráfico. 
- Narrar anécdotas y eventos autobiográficos y biográficos.

- Dar explicaciones, con uso eventual de lenguaje no verbal, en:

- Sustentación de intereses y deseos con razones, causas y efectos.

- Descripciones e instrucciones sobre ubicación, espacio y dirección.

- Instrucciones, reglas y descripciones de juegos y otras actividades de esparcimiento y sociales y de actividades de elaboración de cosas

- Pautas de trabajo académico. (Ordóñez et al, 2008)

Qué preguntas, sobre qué intereses, qué recetas o qué manualidades, qué actividades que necesitan instrucciones, qué noticias, qué mensajes personales y publicitarios, qué anécdotas, qué biografías, qué intereses y deseos, qué sitios, qué juegos, qué reglas, qué actividades sociales y de esparcimiento, qué trabajos de tipo académico y sobre qué temas, todo esto debía ser decidido con colegas y alumnos, así como el lenguaje que valía la pena utilizar y practicar.

En cuarto lugar, los maestros participantes no tuvieron mayor ayuda en sus decisiones pedagógicas al poner en práctica el currículo. No existía en el momento de esta experiencia material ninguno que expusiera explícitamente o diera ejemplos específicos de desempeños auténticos, y no utilizamos material alguno que explicara un currículo cíclico o de naturaleza constructivista; tampoco utilizamos libros de texto para guiar el diseño pedagógico, porque no hay uno que base todo el trabajo presentado en comunicación auténtica que se adapte a alumnos y contextos específicos. Los maestros tuvieron que depender de la guía provista por mí en unas pocas reuniones que me fue posible realizar con ellos durante la experimentación y el estudio mismo, de la que pudieron darles los coordinadores y maestros que habían participado en el grupo de diseño, que eran también novatos en estos cambios, de sus preguntas tanto a ellos como a mí y, sobre todo, de sus propios recursos y valor para atreverse a ensayar.

Finalmente, además de estas exigencias específcas de planeación de clase, del contexto monolingüe donde se movían maestros y alumnos y de los grandes cambios pedagógicos exigidos, las características del currículo exigían de la institución una serie de cambios para permitir que funcionara fluidamente en la práctica, cambios que no fue posible implementar en el año de la investigación en el colegio: en primer lugar, era necesario poner los medios de comunicación, publicaciones y tecnologías de comunicación del colegio al servicio del desarrollo de las lenguas, ya que la autenticidad de la comunicación depende de la existencia de interlocutores, lectores y escritores que tengan motivaciones reales para entrar en interacción; había que rediseñar el sistema de planeación pedagógica, de modo que se pudieran diseñar y poner en práctica desempeños comunicativos que pueden tomar largo tiempo y tiempos variables de desempeño a desempeño; $y$ había que organizar horarios de clases y reuniones de los maestros que facilitaran el trabajo en equipos y las conexiones entre áreas. Lo que lograron hacer los maestros lo lograron sin ninguno de estos soportes.

\section{El estudio}

La investigación aquí presentada comenzó al final de 2008, con los desempeños que ensayaron los maestros antes de que el currículo se pusiera totalmente en uso. Los primeros datos provienen de la información proporcionada por los maestros participantes acerca de estos primeros esfuerzos piloto. En 2009 dejó de usarse el currículo anterior organizado por logros, y entró en pleno funcionamiento el nuevo currículo en las clases de español e inglés de todo el colegio. El estudio completo evaluó cualitativamente el impacto del uso del currículo durante un año. La metodología cualitativa es una forma de evaluar consistente con la naturaleza de los cambios, igualmente cualitativos, que queríamos producir en la enseñanza y el aprendizaje de lengua en el colegio y en la motivación y efectividad del aprendizaje de los estudiantes. Estos cambios debían ser observables en el desempeño de maestros y estudiantes y a través de sus impresiones, opiniones y reflexiones.

Para recoger datos que me permitieran acercarme a las respuestas organicé viajes de campo de jueves y viernes desde Bogotá, en cuatro momentos del 
año académico (febrero, abril, mayo y septiembre), para observar todas las clases de inglés y español que se dictaran en esos días. Tres asistentes de investigación y yo recogimos entrevistas con los maestros y videos de las clases, complementados con entrevistas in situ a los participantes. Escogimos finalmente procesar los datos de todos los maestros de quienes los recogimos completos, que resultaron ser tres maestros colombianos de inglés y seis maestros de español. Transcribimos literalmente los videos y las entrevistas grabadas en audio, e hicimos análisis del discurso de los documentos escritos para categorizar la información.

En el estudio completo buscamos las características de los nuevos ambientes de aprendizaje en ambas lenguas y evidencias de lo aprendido por maestros y alumnos, triangulando resultados entre entrevistas y observaciones. En este artículo uso los mismos datos y análisis para contestar solamente una pregunta específica de investigación: ¿cómo entendieron y llevaron a la práctica los maestros la característica más importante del currículo: los desempeños auténticos? Me concentro en los datos de tres profesores participantes de inglés y dos profesores de español de cuatro niveles diferentes, y construyo con ellos tres casos que ilustran niveles de comprensión diferentes de lo que son desempeños auténticos.

\section{Resultados}

El estudio general produjo evidencia de amplio aprendizaje tanto en profesores como en alumnos, desde este primer año de uso del nuevo currículo (Ordóñez, 2010, 2011; Guevara y Ordóñez, 2011). Los maestros fueron los artífices de un cambio general en los ambientes de aprendizaje de lengua en el colegio, que se convirtieron desde el comienzo del año en escenarios de desempeños comunicativos en las tres áreas de desarrollo del lenguaje indicadas el currículo. Así, en el área de la comunicación cotidiana y mediática, los maestros hicieron que sus alumnos, aun los más pequeños, manejaran como unidad mínima comunicativa intercambios pregunta/respuesta y usaran las preguntas para averiguar información que realmente necesitaban.
Estos intercambios y otros desempeños comunicativos se realizaron también en el contexto de juegos de rol diversos, necesarios cuando se busca la comunicación, sobre todo con niños muy pequeños, para que amplíen su repertorio de uso en contextos imaginados. Los alumnos también desarrollaron y presentaron a sus compañeros álbumes autobiográficos para hacerse conocer, crearon diferentes tipos de programas y noticieros de radio y televisión de su interés y escribieron, pasando por procesos de edición sucesiva, diversos tipos de escritos sociales y artículos periodísticos (Ordóñez, 2010, 2011; Guevara y Ordóñez, 2011).

En el área de aproximación al conocimiento disciplinar los maestros guiaron proyectos de investigación bibliográfica con uso de fuentes diversas, proyectos de investigación empírica basada en la realización de entrevistas y observaciones, presentaciones de conocimiento a audiencias reales y escritura de distintos tipos de textos académicos, también con procesos de edición sucesiva (Ordóñez, 2010, 2011). Y en el área de la comunicación estética y cultural, los alumnos escogieron su literatura preferida, compartieron su lectura con sus compañeros y les recomendaron obras de maneras diferentes, escribieron libretos a partir de obras literarias y originales y los presentaron, y aprendieron, compusieron y cantaron canciones ante audiencias diversas (Ordóñez, 2010, 2011; Guevara y Ordóñez, 2011).

Otro esfuerzo grande de los maestros fue el de adaptar estas ideas generales al nivel e interés de sus respectivos alumnos, de modo que la mayor parte de lo que hicieron en sus clases resulta, además, cumpliendo con una o varias características específicas de autenticidad: el uso de materiales auténticos; la participación estudiantil en las decisiones acerca de qué hacer, sobre qué temas y cómo hacerlo; el trabajo en colaboración, característico en una realidad actual en la que todo conocimiento y todo proyecto o trabajo bien hechos y dirigidos al bien común se logran en grupo y se comunican a otros. Igualmente, los profesores relacionaron lo que pedían hacer a sus alumnos con su propio contexto familiar y social, escolar o de la ciudad en la que viven, y algunos conectaron desempeños de lengua con otros de español, 
inglés y otras áreas académicas, porque hacerlo resultaba natural y surgía del desempeño original (Ordóñez, 2010, 2011; Guevara y Ordóñez, 2011).

Sin embargo, como paso a ilustrar a continuación, la comprensión de los maestros acerca de lo que son desempeños auténticos varía. Para ilustrar distintos niveles de comprensión, tomo ejemplos de tres profesores de inglés que desde el principio demuestran comprensión excelente de lo que es autenticidad comunicativa; uno de español cuya comprensión fluctúa entre excelente en muchos desempeños y no tan buena en varios; y otro de español que solo demuestra comprender muy parcialmente lo que es un desempeño auténtico comunicativo, aunque de todas maneras hace cambios en su clase relacionados con el currículo y positivos para sus alumnos.

\section{Excelente comprensión}

Fueron pocos los profesores de inglés de quienes logramos datos completos en esta investigación, por la rotación permanente que caracterizaba este grupo en el colegio, pero todos ellos demostraron excelente comprensión de la autenticidad en el desempeño comunicativo y lograron adaptarlos a edades bastante diferentes de los estudiantes. Los ejemplos provienen de dos maestras colombianas de grado kínder, con niños de 6 años, y de una, igualmente colombiana, que enseñó en grados $6^{\circ} \mathrm{y}$ $7^{\circ}$ de escuela media.

En grado kínder, las maestras explotaron ampliamente el gusto auténtico de los pequeños por la música y los cuentos para desarrollar desempeños del área estética. Como muestran los siguientes ejemplos, los manejaron sin ninguna reserva, como si fueran en la propia lengua de los pequeños, y los utilizaron auténticamente como juegos y comienzos de conversación que los niños disfrutaban. También conectaron desempeños parecidos en música, lectura comprensiva y producción oral.

Los estudiantes están cantando canciones mientras que hacen mímica relacionada con lo que dicen. Una de las canciones contiene las palabras 'slow' y 'fast.' Los estudiantes mueven las manos según lo señale la canción...En la última parte de la clase la profesora lee a los estudiantes un cuento en inglés haciendo énfasis en palabras que describen acciones como 'fast, quick, slow' y nombres de animales... mostrando los dibujos del libro y usando mímica (Observación de clase - febrero).

Vamos a... leer historias y hacerles preguntas... no preguntas del texto sino ponerlos a imaginar... y hacer la representación de esa historia... Por ejemplo hay una que es sobre el papá que se llama "Singing Dad"... [un] papá [que] canta en el baño, canta en el cuarto, canta en la cocina, "a mi mamá no le gusta y a mí tampoco.” Entonces es preguntarles a ellos... si les sucede o no... (Entrevista - mayo)

A los niños les encanta cantar... Al principio se demoraban más aprendiendo una canción y ahora yo veo que yo planeo un desempeño de canción de hora y media, y cuando ya voy en media hora ellos ya se la saben... hemos sabido escoger canciones sobre las que ellos ya tienen mucho conocimiento previo; entonces al formar la letra de la canción es más fácil... y adquieren mucho más vocabulario porque ya se saben casi todo. (Entrevista - septiembre)

En el área de comunicación diaria, las maestras ayudaron a los niños a pedir información y a darla en interacciones pregunta-respuesta como unidad mínima comunicativa, tal como lo indica el currículo. También les facilitaron situaciones auténticas de búsqueda de información sobre personas desconocidas, por ejemplo llevándoles visitantes al curso.

Hicimos primero... que entre ellos mismos se conocieran utilizando las preguntas y las respuestas que ya les habíamos dado, porque fue al comienzo del periodo y algunos de ellos no se conocían. Luego trajimos personas nuevas para que ellos conocieran, extranjeros, otros profesores bilingües... (Entrevista - mayo)

Los estudiantes hacen una ronda de preguntas en inglés sobre información personal a la profesora que los está visitando, a quien no habían conocido antes; las preguntas se relacionan con el lugar en el que vive, su comida favorita, su deporte favorito, etc. (Observación de clase - febrero) 
Con los pequeños también se comienzan a llevar a cabo desempeños disciplinares. Como se ve a continuación, las profesoras explotaron el hecho de que también enseñan ciencias en inglés y conectan lo que están haciendo en ciencias, con la clase de inglés.

...estuvimos hablando sobre... descripción de personas... características del cabello, de los ojos, de la piel. Nosotros trabajamos con fotos, con ellos mismos, con diapositivas... En ciencias lo estábamos viendo; entonces lo complementamos en inglés. En ciencias ellos veían la parte de aceptación de ellos mismos, de sus compañeros, de interactuar aceptando diferencias... Entonces estuvimos trabajando expresiones para [describir] no solamente el color sino... la forma, el cabello; las personas si eran altas, si eran más pequeñas; entonces ellos comparaban: digamos, 'You are taller than tal persona.' (Entrevista - septiembre)

Incluso al jugar, la situación exigía el uso auténtico de la descripción para identificar personas que se han visto pero no necesariamente se reconocen.

Los estudiantes se organizan en parejas para trabajar con unas fichas que realizaron previamente, con recortes y dibujos con caras de personas. El propósito de la actividad es jugar 'Guess who. Uno de los estudiantes debe escoger una de las fotografías y dar las características físicas de esta persona a su compañero, quien debe adivinar quién es el personaje. La profesora pasa por cada grupo revisando lo que están haciendo los estudiantes y participa también en el juego. También se hace con los mismos estudiantes. Se escogen cinco para que pasen al frente y luego otro estudiante comienza a dar pistas a otro compañero para que adivine de cuál de los cinco está hablando. (Observación de clase - septiembre)

Las profesoras de estos pequeños explotaron desempeños que auténticamente entusiasmaron a los niños, para apoyarlos en el uso de una enorme cantidad de lenguaje. Extendieron desempeños preferidos de los niños a más de un período escolar, siguiendo el currículo cíclico, con avances ostensibles en el lenguaje de los niños. Un ejemplo es el del desempeño del día a día de describir lo que hacen las personas según su profesión. Los niños comenzaron involucrándose totalmente en el trabajo de sus padres.

...Cada estudiante había dibujado a sus padres y las profesiones que practicaban; todos los dibujos se ubicaron en una parte del salón a la que llamaron "The professions wall." Un estudiante pasa al frente, escoge un dibujo de otro compañero y comienza a preguntarle sobre las profesiones de sus padres según el dibujo (todo en inglés)... La profesora corrige los errores de los estudiantes. Preguntan sobre el nombre de los padres, la profesión, el lugar donde trabajan...

En clase:

Profesora: So, who wants to go to the professions wall and present. XXX... Ok... Remember... to talk aloud.

Est1: Whose picture is this?

Est2: Mine!

Est1: What's your father's name?

Est2: Carlos Alberto.

Est1: Your father's name is Carlos Alberto. What's your father profession?

Est2: Doctor.

Est1: Your father profession is doctor. She works in a...

Est1: In the hospital.

Profesora: Juan Pablo, who works in the hospital? Est1: The father... the father Juan Andrés.

Profesora: Juan Andrés' father.

Est1: Juan Andrés' father.

Profesora: How, how do you say that, sorry? He or she?

Est1: He.

Profesora: Ok... (Observación de clase - abril y mayo)

Desempeños como los relacionados con las profesiones siguieron ampliándose con uso de más lenguaje en juegos de rol, que los mismos niños hicieron más auténticos con sus propias ideas.

...En clase a veces... ellos mismos [los niños] dicen cosas y... hacemos cosas que no estaban en la planeación...; o de pronto las clases planeadas funcionaron, pero pudimos ver que ellos querían más; entonces les añadimos más y estamos ano- 
tando todo en nuestras planeaciones para cuando volvamos a hacerlo... Hay desempeños como el de profesiones que se repiten a medida que transcurre el año... sobre profesiones de un hotel queríamos llevarlos a un hotel. Ya estábamos averiguando en un hotel donde hay guía bilingüe..., pero ellos dijeron: "No, pero si aquí mismo [en el colegio] tenemos algunas profesiones del hotel. Vamos a ver a la recepcionista..." Y ellos mismos dieron la idea y nos fuimos a hacer el recorrido [por el colegio] hablando, preguntándole cosas a la recepcionista, al vigilante... (Entrevista - mayo)

Los estudiantes están jugando al doctor... parte del desempeño de profesiones. Los estudiantes tienen diferentes roles como médicos, recepcionistas, enfermeros y pacientes. Están divididos en grupos y utilizan juguetes para simular una situación en la que alguien va al médico. Utilizan expresiones como "How can I help you? I need a doctor. What's your name? What is your telephone number? What's your profession? My baby is ill. Let me see the stomach, the eyes, etc.... (Observación de clase - mayo)

...Ellos hacen juegos con las profesiones... La primera vez que lo hicimos, hablamos de las profesiones de los papás y las mamás. Entonces hicimos un listado de las profesiones que había, y ellos trajeron vestuario para representar. Pues obviamente ellos ya sabían cómo había sido su experiencia, por ejemplo cuando van al doctor... y la usamos para decidir qué aprender a decir... y los niños lo disfrutan mucho. (Entrevista septiembre)

Y la explotación de la descripción de actividades profesionales y desempeños asociados con ellas continuó durante el año, en diferentes versiones, con el uso de lo que ocurre en el contexto de los niños y de los medios a su alcance.

Vino una chef internacional al colegio; entonces [los niños] estaban súper animados. Ella vino, cocinó, mostró un video de cuando salía en televisión... Dijimos pues, ¡aprovechemos!... Unos eran asistentes, otros eran presentadores. Vimos unos shows de cocina, hablamos de unos elementos [de cocina], de comida, trajimos libros de recetas... hablamos de recetas, después habla- mos de pasos... muy simples como cortar, como poner en el horno.... A ellos les gusta porque ellos cuentan que allá en sus casas ellos le ayudan a sus papás... que ellos mismos, digamos, abren la nevera y sacan tal cosa, hacen un Chocolisto... que... necesita leche... chocolate, una cuchara... (Entrevista - septiembre)

En clase:

Los estudiantes están haciendo una presentación sobre recetas. Los niños se organizaron en grupos de tres: el primero presenta su grupo y la receta que van a preparar, el siguiente presenta los ingredientes y los utensilios que van a utilizar y el último estudiante prepara y describe la receta (todo en inglés). Cada estudiante se vistió de acuerdo a su papel y además llevaron a la clase los ingredientes y los materiales que iban a utilizar. (Observación de clase - septiembre)

En los grados $6^{\circ}$ y $7^{\circ}$ era mucho más fácil lograr autenticidad, por la edad más avanzada de los niños y niñas, con repertorios de lenguaje y posibilidades de uso mucho más amplias que en los pequeños. La profesora que dirigió el inglés de estos grados lo hizo con mucha seguridad. Desde el principio y a lo largo del año, por ejemplo, trabajó investigaciones de diferentes tipos con los niños, con productos igualmente diferentes tanto de tipo académico como mediático, que fueran del interés de ellos. Al permitirles participar completamente en las decisiones sobre sus investigaciones, con su guía, tuvo en cuenta la autenticidad del quehacer de investigar, que lleva a quien lo hace por caminos diferentes cada vez a medida que avanza en su exploración.

Vamos a terminar un magazín sobre un tema que escogieron ellos. En cada clase tienen una agenda que desarrollar. Lo primero que hicieron fue preguntas sobre lo que les gustaría investigar sobre ese tema que escogieron... Con base en las preguntas, desde antier se les pidió que trajeran información que podían consultar por Internet, por libros, por revistas, no importaba si era en español o en inglés, para que aquí empezaran a discutir y a responder esas preguntas...Por ejemplo, ese grupo... iniciaron solamente con que querían saber qué era una comedia y ya; pero ahorita... quieren 
comparar películas y de ahí sacar la información y las características de qué es una comedia. A mí eso me parece interesantísimo, (así que les dije): 'Miren las preguntas que tienen y piensen si les sirven realmente para lo que quieren hacer'. Y llegaron a la conclusión de que tenían que cambiar sus preguntas. (Observación de clase - febrero)

A pesar de su juventud, estos niños comenzaron a hacer investigación empírica, con todos los elementos que la caracterizan. En el siguiente ejemplo se ve esto y se aprecia la forma como la profesora trabaja el lenguaje que aparece naturalmente como importante, en un desempeño que ella considera claramente auténtico.

[Lo] que estamos haciendo ahorita es "research." La primera fase es la identificación de un problema. Yo les dije a ellos que podían tomar... problemas del colegio o de su [barrio] o del lugar donde viven, o que si quieren arriesgarse a hacer algo sobre la ciudad tienen la libertad de hacerlo. Entonces esa primera fase es cómo observar, indagar, pensar en [para] qué cosas o qué situaciones a ellos les gustaría encontrar la respuesta... [Luego] formulan la pregunta de investigación y escogen [los participantes]... Después de eso pues van a trabajar sobre preguntas que les puedan ayudar... a planear las entrevistas, las encuestas, y en ese momento la idea es que revisemos un poco la estructuración de preguntas...Van a interrogar a la gente que tengan que interrogar y luego, a la par, van a estar revisando lo que han escrito otros sobre ese tema... Eso también los obliga a ellos a ir a fuentes bibliográficas. Después de eso van a analizar resultados, trabajar por categorías de información, qué encontraron para responder sus preguntas. También hay un momento de hacer hipótesis, de usar condicionales, van a pensar en posibles respuestas a sus preguntas y luego la idea es analizar las respuestas y concluir y presentar esos resultados...

...Considero que este es un desempeño auténtico... porque en la vida real tú... estás haciendo ese ejercicio de cuestionarte e indagar y encontrar respuestas a esos cuestionamientos. En la universidad y en el colegio necesitas ir a [fuentes] y hasta en tu vida profesional, siempre necesitas estar le-
Educación para el bilingüismo y aprendizaje de maestros: comprensión del desempeño auténtico en la acción de cambiar prácticas pedagógicas
Claudia Lucía Ordónez

yendo y estar consultando y pienso que esa es una habilidad que hay que trabajar en estos muchachos para que dejen de ver la investigación como una cosa de bata y laboratorio... que entiendan que la razón de ser de nosotros como profesionales es... analiza(r) problemas, busca(r)... y el día que uno deje de hacer eso... se fosilizó. (Entrevista septiembre)

En el área estética, la maestra manejó la libertad con que los muchachos deben escoger qué leer y qué hacer con lo que leen y su propia experiencia, y los guió para que actuaran como autores. Escribir creativamente en una lengua extranjera no es un desempeño muy auténtico, porque no es probable que una persona con una lengua nativa consolidada decida expresarse en una extranjera. Pero la forma como la maestra maneja la producción de historias hace auténtica no solo su escritura, con ediciones sucesivas y para una audiencia específica, sino la lectura y comprensión de otras obras, la lectura oral, el uso y la evaluación del producto.

Ellos elaboraron unos cuentos para los niños de preescolar, lindísimos... y tuvimos un espacio para sentarnos a leer[les] esos mismos cuentos y... escuchar si les habían gustado o no... Hubo una fase de leer otros cuentos [para niños]... y de recordar ellos cuentos que les habían llamado la atención cuando eran niños. Fueron a la biblioteca y escogieron temas que quisieran desarrollar en esas historias. Cuando estaban en la parte de exploración, indicaron los elementos que tenían los cuentos... y los analizaron para incluirlos en el cuento que ellos iban a elaborar... Después... desarrollaron los cuentos con diferentes valores... hicieron un borrador... se sentaron con compañeros para leer el cuento. Luego yo lo revisé, hice correcciones... Hicieron el proceso de edición y de revisión, y luego ensayaron entre ellos cómo contar las historias... y luego fueron a preescolar... [Luego] nos sentamos a evaluar como habían sido, cuales habían sido sus fortalezas, qué cosas hay que mejorar, y fue interesante. (Entrevista septiembre)

Un caso parecido en el que la maestra dirigió un proceso de convertir una actividad de lengua en un desempeño auténtico, puede verse en este 
sencillo ejemplo en el que los alumnos aprendieron a dar instrucciones en un contexto cotidiano para ellos, y las revisaron contra la forma como otros las entendieron y las pudieron seguir: "Con ellos hicimos texto instructivo, que era diseñar unos juegos y [escribirles] sus propias instrucciones. Los jugaban entre ellos, los escribían, los mostraron a otros niños..." (Entrevista - septiembre)

\section{Comprensión fluctuante}

Es en los grados iniciales de preescolar y primaria, en el área de español, donde aparecen actividades pedagógicas que parecen todavía no haberse desprendido de usos anteriores. En grado $2^{\circ}$, la maestra de español lleva a su salón de clase varios desempeños con buenos rasgos de autenticidad, pero cae también en ocasiones en la actividad divertida pero poco auténtica para sus niños. Comenzando por los desempeños que se acercan a la autenticidad, el siguiente muestra cómo la maestra utiliza el interés de los estudiantes sobre sí mismos para que comuniquen sus experiencias e información personal a otros. También los guía para conseguir información de fuentes primarias, sus padres, a pesar de que la forma como lo hace parece compleja y poco natural. La maestra usa el trabajo en colaboración y utiliza la comunicación para practicar la construcción de preguntas.

Este desempeño se llama 'Mis mejores recuerdos'... Empezamos haciendo preguntas... ellos no saben las respuestas, pero les van a decir a los papitos: ¿Cuándo nací yo? ¿Cómo vine al mundo?... Tiene como propósito también la expresión de sentimientos y que pudieran construir las preguntas utilizando las palabras claves con los signos de interrogación... Ellos están elaborando un álbum... de recuerdos... En cada clase se dan cinco o diez minutos para ir reconstruyendo el álbum y las fotos que [traen]. Hoy... están haciendo preguntas sobre lo que pasa ahí. Van a narrar y después van... a elaborar párrafos descriptivos (sic) donde cuenten lo que [está pasando] en la foto, pero ya... enriquecido por las preguntas que les van a hacer los compañeros... La intención es que, cuando ellos ya lo terminen, podamos invitar a los papitos a que escuchen lo que ellos piensan, $y$ [ahí] les van a dar las respuestas a esas preguntas... y obviamente los papitos también podrán [hacer] preguntas ese día... (Entrevista - febrero)

En clase:

Profesora: ...Voy a dejarlos libremente en esta ocasión... tú vas a poderte hacer con los amigos que tú quieras... Nos vamos a hacer con tres compañeros más ...Tú les vas a contar a tus amigos qué es lo que pasa en cada una de esas fotografías, con quién estás, dónde estás, o sea, vamos a utilizar ¿qué?

Todos: Las palabras... claves.

Profesora: ¿Para qué? Para hacer preguntas... Primero tú vas a dejar que tu compañero te cuente todo lo que quiera contarte... en qué lugar estás, con quién estás, qué está pasando... Entonces si él no ha dicho algo, tú puedes pedir la palabra y formularle la pregunta. (Observación de clase febrero)

En otro desempeño, que tiene el propósito de practicar la descripción de animales, la maestra logra encontrar una situación imaginaria en que sería totalmente comunicativo para los niños describir a su mascota, pensando rigurosamente en la información que mejor permitiría a otros identificarla: la mascota se perdió. Es, sin embargo, solo el final de una sección de su clase en la que trabajaron las mascotas como tema.

Acaban de finalizar... 'Valorando y buscando mi mascota'... [un desempeño en el que] terminaron elaborando afiches donde ponían la información de un animalito que se les perdió. Entonces hablamos de las mascotas, ampliamos el mundo de los animales y finalizaron [con] un afiche de la supuesta pérdida de su mascota con la información que tenían para encontrar[la]... En este caso estamos trabajando la descripción y se hace a la par con el trabajo que se hace de composición. (Observación de clase - febrero)

La maestra se acerca más a la autenticidad del nivel de los niños, en un desempeño de desarrollo de un noticiero escolar. Ella les indica cómo hacerlo, pero los niños piensan en noticias, interesantes 
para ellos, de su contexto escolar, después de ver y comentar un noticiero real.

[Primero]... una lluvia de ideas... donde los niños comunicaron y expresaron sus gustos, sus preferencias frente a los programas de televisión. Cuando llegan a... lo que no les gusta, llegan al noticiero... [Dicen] que es porque ahí presentan cosas muy feas para los niños, porque los papás no les dejan ver noticias, porque a esa hora ya están durmiendo o están haciendo otro tipo de actividades... La propuesta era [hacer] un noticiero... que nos interese... de cosas que pasan en nuestro medio... Cada estudiante debía escribir una noticia sobre algún evento que haya ocurrido en el colegio... Leyeron sus noticias y recibieron comentarios de sus compañeros. [Luego]... fueron a la sala de informática para ver una parte de un noticiero real... donde pudiéramos observar cuál es el vocabulario que se utiliza, qué tipo de noticias se presentan, cómo las presentan, qué tanto tiempo demora una noticia de esas... Ya después, con esa información, ellos pueden volver a la noticia y hacerle algún arreglo. Ahí vamos en este momento...

[Luego] los organizo por grupos para ya empezar a trabajar la noticia... Buscarán las fuentes [para] hacer la recolección de la información, las entrevistas... Luego empezamos la redacción de la noticia... ya con el acompañamiento del profesor. Una vez las tengan en limpio, ya empezamos a hacer lo que es la puesta en escena de manejo vocal, de manejo de expresión oral, para ya empezar a montar el noticiero y presentárselo a los compañeros, y si se decide,... invitar a los papás también a que escuchen el montaje de un noticiero (Observación de clase y entrevista - septiembre)

La maestra toma otra acertada decisión comunicativa al practicar la lectura comprensiva con sus alumnos, por medio del manual de convivencia del colegio, información escrita que los afecta y que necesitan conocer. Sus palabras, sin embargo, parecen indicar que la lectura y la escritura no son para ella ocasiones de comunicación.

El desempeño era leer textos informativos para luego hacer comprensión, respondiendo a diferentes niveles de significado. En este caso teníamos que velar porque los niños tuvieran que consultar, leer, socializar información... Entonces escogimos... conocer [sus] deberes y derechos [en el colegio]. Cogimos el mismo manual de convivencia porque necesitábamos que ellos hicieran lectura de ese manual, pero [que] a la vez hicieran comprensión e interpretación de eso que ellos leían y a su vez encontraran la razón de ser. Obviamente, no solamente nos quedamos en la comunicación; damos mayor relevancia a la comunicación pero siempre va acompañado de un proceso de producción escrita. (Entrevista septiembre)

En las siguientes descripciones rápidas que la maestra hace de desempeños que ha llevado a cabo en su clase, mezcla muchas cosas que aparecen en el currículo, sin claridad ninguna sobre la forma como constituyen desempeños auténticos comunicativos.

...Trabajamos... con rondas, fonomímicas, adivinanzas, y pues obviamente ahí, dentro de los desempeños continuos, aparece nuevamente el hacer preguntas, contestar preguntas, escuchar y participar en situaciones de comunicación, de conversación, continuar el proceso de lectura y de escritura, tanto en la lectura de las canciones y poemas pero también en la producción misma...

En este [desempeño disciplinar], 'Pequeños informantes..., ellos van a informar sobre su música favorita, cuál es el cantante que más se destaca, a qué género pertenece... Van a sala de informática, consultan en los libros, en biblioteca y luego hay... espacios para socializar esa información con los compañeros. Hacen expresión de juicios y diferencias, vuelve el hacer y contestar preguntas, vuelven a aparecer desempeños continuos que son recurrentes, como proponer actividades y argumentar su realización oral. Entonces son ellos los que dicen cómo lo van a presentar a sus compañeros, bien sea [por medio de] una exposición que hagan, mediante un folleto... Luego ellos lo presentan a los compañeritos y luego viene el proceso de hacer y responder preguntas de acuerdo a su interés. (Entrevista - septiembre)

Finalmente, hay desempeños organizados por esta maestra que fallan totalmente en el área de la autenticidad. El siguiente, por ejemplo, es divertido 
para los niños pero busca una manera extraña y artificial de practicar la construcción de preguntas, que resulta nada comunicativa y poco comprensible para los niños. Las preguntas funcionan solo como estructuras gramaticales y no como formas auténticas de averiguar información desconocida y necesaria.

Los estudiantes están escuchando la canción 'La gallinita Josefina'. Pueden cantar y bailar al tiempo que oyen. Deben identificar información de la canción que puede ser respuesta a una pregunta, y formular la pregunta correspondiente a sus compañeros. Oyen la canción, la maestra da un ejemplo de preguntas que se pueden hacer sobre el cuento que hay en ella (Canción: "La gallinita Josefina, puso un huevo en la cocina...” ¿Dónde puso el huevo?") y los niños ensayan, con trabajo. Resulta un ejercicio bastante complicado que no parecen entender muy bien. (Observación de clase - abril)

\section{Comprensión incompleta}

De nuevo en los cursos de los alumnos pequeños, en este caso transición y grado $1^{\circ}$, la profesora de español trató de desarrollar actividades con algunas características de los desempeños auténticos, especialmente la participación de los estudiantes con sus intereses, pero no llegó a la autenticidad comunicativa. Los niños, sin embargo, siempre se mostraron interesados y entusiastas en su clase, involucrados en temas que les interesaban y participando con entusiasmo. En los primeros ejemplos, que corresponden a los últimos datos que tomamos durante el año de la investigación, hay algo de significado. Primero, en el contexto del tema 'mi fiesta favorita', y no de una situación de comunicación auténtica real o imaginada, la profesora lleva a los niños a que produzcan preguntas y expliquen su tema fiestero preferido.

Nosotros escogimos el tema 'mi fiesta favorita'... Se realizaban preguntas, y también se daba la oportunidad a los niños de que sustentaran la respuesta de por qué les gustaba esa piñata... que sustentaran sus gustos... Los niños tenían la oportunidad de opinar... Pasaban al frente, realizaban preguntas y sustentaban... por qué querían que su piñata la hicieran de 'Power Rangers' y por qué no de otra cosa. (Entrevista - septiembre)

Los propósitos de aprender a hacer preguntas y a sustentar opiniones son importantes y son parte del currículo, pero no es claro que la maestra encuentre formas en las cuales se justifique comunicativamente que se lleve a cabo esta discusión. Esto hubiera sido posible, por ejemplo, si los niños hubieran organizado una verdadera fiesta para alguna ocasión auténtica.

En el siguiente ejemplo, aun cuando la maestra trabaja lectura de cuentos, una de las actividades favoritas de los niños, su trabajo parece mecánico y poco concentrado en el significado que la lectura pueda tener para los niños, relacionándola con sus contextos e intereses. Hay predicción de la historia con base en los dibujos que la acompañan; de resto, solamente memoria sobre información explícita.

En esta clase la profesora lee un cuento a los estudiantes y a medida que van leyendo, pide a los estudiantes que repitan información que aparece en el cuento. Algunas veces la docente deja que los estudiantes sugieran lo que va a pasar en el cuento de acuerdo con los dibujos que están en el libro.

(Observación de clase - septiembre)

Durante el año, sin embargo, los niños hacen muchas cosas pero no es claro que estén comunicándose ni qué, con quién o para qué lo hacen. La profesora organiza sus actividades enfocada en los temas a tratar, inclusive llama a los temas de conversación desempeños, y se concentra en las formas lingüísticas que debe trabajar, pero no logra relacionar esas formas con el propósito de la comunicación, con quiénes se comunican, lo que se comunica y/o la situación en la cual ocurre la comunicación.

Les di la oportunidad de que ellos me dijeran qué era lo que querían conocer. Entonces en el primer periodo hablaron sobre el universo; querían saber qué eran los meteoritos, los planetas. Sacamos 26 temas e hicimos votación, y al final sacamos cuatro temas que son: los meteoritos, los volcanes, los dinosaurios y los cocodrilos. Entonces el salón se dividió en cuatro grupos y empezamos a trabajar en esos desempeños [i? ?. Todas las fases se 
ejecutaban... fases de preguntas, de lo que a ellos les gustaba [saber]... En primero estamos viendo lo que es estructurar una oración; entonces el desempeño gira alrededor de cómo ellos estructuran la oración para llevarlos a que hagan un párrafo a final de año. (Entrevista - mayo)

Los mismos elementos aparecen en la siguiente actividad, en la que de nuevo no es clara la intención de comunicar algo específico a alguien, para algún propósito auténtico. El proceso de lectoescritura parece continuar basándose en actividades de tipo mecánico.

A cada uno se le dio una guía donde ve los muñequitos, va a colorear, los va a recortar, los pega en el cuaderno y después, con estos muñecos, [van] a formar oraciones... Como ellos están en escritura espontánea, formamos las oraciones, la profesora las escribe en el tablero, las leemos entre todos, miramos cuántos golpes hay por cada palabra y después ellos la escriben como puedan en el cuaderno... (Observación de clase - mayo)

Inclusive cuando la maestra usa una ocasión de comunicación auténtica como la escritura de tarjetas para el día del amor y la amistad, de nuevo hacia el final del año, no es claro cómo funcionan estos mensajes como comunicación y expresión de significado; la maestra menciona descripciones, pero no se entiende qué tienen que ver con las tarjetas; y se concentra de nuevo en la forma y no en el significado ni en la función de las tarjetas.

...Ahorita que hicimos lo del amor y la amistad... como hasta tercer periodo de primero se ve estructuración de oraciones, entonces realizamos descripciones y ellos tenían que escoger una persona, la que ellos quisieran, para realizar una tarjeta... Entonces unos escogieron a los papás, otros a los profes, otro a la mascota, mi muñeco favorito, otros a la niñera... (Entrevista - septiembre)

\section{Discusión}

Este año de trabajo en el cambio pedagógico real de la práctica, constituyó para mí el mejor programa de desarrollo profesional que he dirigido en muchos años de dedicación a esta labor, por sus efectos en los maestros, en los alumnos y en el colegio. La puesta en marcha del currículo les exigió a los profesores, desde el principio, un enorme trabajo de creación, puesta en práctica y autoevaluación de sus ideas y acciones, en medio de dudas y con poca ayuda. Lo hicieron de manera ejemplar y lograron cambiar las características de los ambientes de aprendizaje de lenguas en toda la institución, así como la motivación y forma de usar tanto el español como el inglés de sus alumnos.

El valor del cambio se ve intensificado por el hecho de que se logró, no a partir de largos cursos de formación o entrenamiento específico, sino solamente con base en la apertura y disposición de los maestros hacia el cambio y su aceptación de la inquietante responsabilidad de lanzarse a la acción. Desde luego, y como lo indica la descripción del aprendizaje humano que hace el constructivismo, cada maestro parte de lugares diferentes de experiencia, conocimientos y convicciones anteriores, y logra comprensiones de diferente nivel acerca de lo nuevo que se quiere poner en práctica. Esto es lo que demuestran los datos de este estudio, a pesar de que todos los maestros se aproximan, definitivamente, a muchas de las características de diseño de desempeños que el currículo indica. Parece ser más difícil, para los maestros de niños muy pequeños, desprenderse de la concepción de actividad de salón de clase que han utilizado y que han desarrollado sobre todo alrededor de temas que consideran adecuados para sus alumnos. Algunos todavía no logran separarse completamente de sus concepciones anteriores sobre lo formal, lo estructural del lenguaje y a veces lo mecánico de las habilidades formales escolares que se han utilizado tradicionalmente para lograr, por ejemplo, el aprendizaje inicial de la lectura y la escritura. Es así como se presentan casos de comprensión parcial y no tan buena de lo que es un desempeño auténtico comunicativo.

Sin embargo, hay casos de comprensión excelente, mucho más numerosos que los de comprensión parcial o errada, y hay cambio generalizado en el colegio (Ordóñez, 2010, 2011; Guevara y Ordóñez, 2011). Realmente todos los maestros cambiaron en alguna medida sus usos pedagógicos anteriores, 
utilizando el trabajo en colaboración y los intereses y aportes de sus alumnos. Los logros son múltiples, a pesar de que los maestros no dejaron hasta el final de expresar dudas e inseguridades, naturales en personas que con tanto valor se lanzaron a un cambio radical sin el apoyo de libros de texto, modelos o descripciones detalladas de lo que debían hacer. Seguramente están capacitados para ayudarse más aún entre ellos y ayudar a otros a mejorar e iniciar sus propios cambios, y avanzarán aún más si siguen trabajando y si lo hacen en colaboración con sus colegas.

No hay que olvidar que este año fue apenas el primero de uso del currículo, de modo que estos resultados son sorprendentes en un proceso de cambio total de concepciones y prácticas pedagógicas. Y falta mucho por hacer. El colegio, como cualquier institución educativa de verdad interesada en cambiar sus prácticas pedagógicas y aumentar la calidad de la formación de sus estudiantes, debe sustentar de manera decidida el trabajo de sus maestros. Ellos son los artífices del éxito. En relación con este currículo en particular, debe estimular y facilitar la continuación de la experiencia; debe llevar a la realidad las exigencias administrativas y de organización que hace el currículo, para facilitar períodos extendidos de trabajo de los alumnos y reuniones de planeación de maestros de las dos áreas de lengua; y debe convertir los medios de comunicación, audiovisuales $y$ virtuales del colegio en verdaderos recursos de comunicación de la comunidad educativa, manejados por los estudiantes con la guía de sus maestros y dedicados al desarrollo auténtico de las lenguas.

Debo hacer énfasis en el trabajo colegiado de cambio en la práctica como forma exitosa de desarrollo profesional. Dependemos demasiado de cursos de formación y prédicas de cambio en la formación inicial de maestros y, sobre todo, en el desarrollo profesional de maestros en ejercicio, y los cambios en educación continúan siendo difíciles y a menudo inexistentes. Una comunidad entera de maestros enfrentada a la necesidad inmediata de producir prácticas diferentes puede lograr -trabajando junta- cambios rápidos y sorprendentes. Aprender en la acción es uno de los más importantes principios constructivistas, y puede producir extraordinarios resultados cuando se pone en práctica acompañado de tiempo para que ocurran los procesos, trabajo en colaboración y apoyo, este último no demasiado elaborado. Vale la pena ampliar el uso de este tipo de programa con propósitos de desarrollo profesional para maestros. Y la búsqueda de una verdadera y efectiva educación para el bilingüismo es un propósito educativo importante hoy en día, que puede proporcionar un contexto ideal para este tipo de formación.

\section{Referencias}

Ausubel, D. (1968). Educational Psychology: A Cognitive View. New York: Holt, Rinehart, and Winston.

Barriga-Villanueva, R. (2002). Estudios sobre el habla infantil en los años escolares: "...un solecito calientote”. México: El Colegio de México.

Barriga-Villanueva, R. (2003). Construyendo realidades. El lenguaje infantil de los años escolares. En E. Matute y F. Leal (Eds.), Introducción al estudio del español desde una perspectiva multidisciplinaria (pp. 183-218). Guadalajara: Universidad de Guadalajara.

Boix-Mansilla, V. \& Gardner, H. (1998). What are the Qualities of Understanding? En M. Stone-Wiske (Ed.), Teaching for Understanding: Linking Research with Practice (pp. 161-196). San Francisco: JosseyBass Publishers.

Brill, J. (2001). Situated cognition. En M. Orey (Ed.), Emerging Perspectives on Learning, Teaching, and Technology. Documento en línea. Recuperado el 3 de octubre de 2011. Disponible en http://projects. coe.uga.edu/epltt/

Brown, J., Collins, A. \& Duguid, P. (1989). Situated Cognition and the Culture of Learning. In Educational Researcher, 18(1), 32-42.

Bruner, J. (1960). El proceso de la educación. México: Manuales UTEHA, $\mathrm{N}^{\circ} 181$.

De Mejía, A., Ordóñez, C. y Fonseca, L. (2006). Estudio investigativo sobre el estado actual de la educación bilingüe (inglés-español) en Colombia. Informe de investigación inédito. Bogotá: Ministerio de Educación Nacional de Colombia y Universidad de los Andes.

Dewey, J. (1945). Experiencia y educación. (Trad. Lorenzo Luzuriaga). Buenos Aires: Editorial Losada S.A.

Dewey, J. (1948). El niño y el programa escolar. (Trad. Lorenzo Luzuriaga). Buenos Aires: Editorial Losada S.A. 
Díaz Barriga, F. (2003). Cognición situada y estrategias para el aprendizaje significativo. En Revista Electrónica de Investigación Educativa, 5(2). Documento en línea. Recuperado el 12 de julio de 2010. Disponible en http://redie.ens.uabc.mx/vol5no2/contenidoarceo.html

Gardner, H. (2004). La educación de la mente y el conocimiento de la disciplinas. Barcelona: Ediciones Paidós Ibérica, S.A.

Guevara, D. y Ordóñez, C. (2011). Impacto de la enseñanza del inglés por medio de desempeños auténticos a estudiantes de grado kínder de un colegio de Ibagué. En Memorias del Encuentro Nacional de Investigación y Desarrollos ENID 2011. Bogotá: Universidad Nacional de Colombia.

Insuasty, E. (2008). The Place of Authentic Materials in Language Teaching: A Historical Perspective. In ASOCOPI Newsletter, October, 8-9.

Lave, J. (1997). The Culture of Acquisition and the Practice of Understanding. En D. Kirschner \& J. Whitson (Eds.), Situated Cognition: Social, Semiotic, and Psychological Perspectives. (pp. 17-35). Mahwah, NJ: Lawrence Erlbaum Associates.

Ninio, A. \& Snow, C. (1996). Pragmatic Development. Boulder, CO: Westview Press.

Ordóñez, C. (2000). Oral Bilingual Proficiency of Colombian Adolescents. Tesis doctoral inédita. Cambridge, MA: Escuela de Educación, Universidad de Harvard.

Ordóñez, C. (2004a). EFL and Native Spanish in Elite Bilingual Schools in Colombia: A First Look at Bilingual Adolescent Frog Stories. In International Journal of Bilingual Education and Bilingualism, Special Issue: Bilingual Education in South America, 7(5), 449-474.

Ordóñez, C. (2004b). Pensar pedagógicamente desde el constructivismo. De las concepciones a las prácticas pedagógicas. En Revista de Estudios Sociales, 19, 7-12.

Ordóñez, C. (2005). Oral Bilingual Proficiency of Colombian Adolescents in an Elite Bilingual School. En J. Cohen, K. McAllister, K. Rolstad \& J. MacSwan (Eds.), ISB4: Proceedings of the Fourth International Symposium on Bilingualism (1765-1783). Somerville, MA: Cascadilla Press. Documento en línea. Recuperado el 3 de octubre de 2011. Disponible en http:// www.cascadilla.com/isb4.html

Ordóñez, C. (Comp.). (2007). Memorias del Segundo Simposio de Bilingüismo y Educación Bilingüe en América Latina. Bogotá: Ediciones Uniandes.
Educación para el bilingüismo y aprendizaje de maestros: comprensión del desempeño auténtico en la acción de cambiar prácticas pedagógicas

Ordóñez, C. (2007). Introduction. En C. Ordóñez (Comp.), Memorias del Segundo Simposio Latinoamericano de Bilingüismo y Educación Bilingüe en América Latina (pp. I-II). Bogotá: Ediciones Uniandes.

Ordóñez, C. (2008). Education for Bilingualism in International Languages in a Monolingual Socio-Linguistic Context. En Lenguaje, Vol.36 (No.2), 253-384.

Ordóñez, C. (2010a). Desempeño auténtico en educación. En C. Ordóñez y C. Castaño, Pedagogía y didáctica: Texto del docente (pp. 151-160). Quito: Ministerio de Educación de Ecuador.

Ordóñez, C. (2010b). Educación para el bilingüismo en contexto monolingüe: dos lenguas conectadas desde el currículo. En Estudios de lingüística inglesa aplicada ELIA, 10, 41-76.

Ordóñez, C. (2011). Education for Bilingualism: Connecting Spanish and English from the Curriculum, into the Classroom, and Beyond. In PROFILE, 13 (2), 147-161.

Ordóñez, C., Bonilla, A., Arias, I., Casallas, J., Barrero, A. y Lastra, S. (2008). Currículo conectado de español e inglés para el Colegio Bilingüe San Bonifacio de las Lanzas. Documento inédito. Ibagué: Colegio San Bonifacio de las Lanzas.

Perkins, D. (1993). Teaching for Understanding. In American Educator: The Professional Journal of the American Federation of Teachers, Vol. 17 (No. 3), 8, 28-35.

Perkins, D. (1998). What is Understanding? En M. StoneWiske (Ed.), Teaching for Understanding: Linking Research with Practice (pp. 39-57). San Francisco: Jossey-Bass Publishers.

Perkins, D. (2005). La enseñanza para la comprensión: cómo ir de lo salvaje a lo domesticado. En Revista Internacional Magisterio, 14, 11-13.

Perrone, V. (1998). Why do we need a Pedagogy of Understanding? En M. Stone-Wiske (Ed.), Teaching for Understanding: Linking Research with Practice (pp. 13-38). San Francisco: Jossey-Bass Publishers.

Piaget, J. (1970). Piaget's Theory. En P. Mussen (Ed.), Carmichael's Manual of Child Psychology, Vol. 1. New York: Wiley.

Piaget, J. (1999). De la Pedagogía. Buenos Aires: Editorial Paidós.

Savery, J. \& Duffy, T. (1996). Problem Based Learning: An Instructional Model and its Constructivist Framework. En B. Wilson (Ed.), Constructivist Learning Environments: Case Studies in Instructional Design 
(pp. 135-147). Englewood Cliffs, New Jersey: Educational Technology Publications, Inc.

Snow, C. (2007). Determinants of the Outcomes of Language Education: Developmental and Sociolinguistic Factors. En C. Ordóñez (Comp.), Memorias del Segundo Simposio Latinoamericano de Bilingüismo y Educación Bilingüe en América Latina (pp. 2-12). Bogotá: Ediciones Uniandes.

Stone-Wiske, M. (1998). What is Teaching for Understanding? En M. Stone-Wiske (Ed.), Teaching for Understanding: Linking Research with Practice (pp. 61-86). San Francisco: Jossey-Bass Publishers.

Van Lier, L. (2005). Interaction in the Language Curriculum: Awareness, Autonomy, and Authenticity. Londres: Longman.

Vygotsky, L. (1978). Mind in society. Cambridge, MA: Harvard University Press.

Widdowson, H. (1978). Teaching Language as Communication. Oxford: Oxford University Press. 